\section{Opening a door into solid tumors}

\section{By Tim Fulmer, Staff Writer}

The irregular and leaky vasculature of tumors has been a major obstacle to the effective delivery of chemotherapeutics and immunotherapies to the cancerous tissues they target. ${ }^{1}$ A paper in Nature now suggests that inhibiting regulator of $\mathrm{G}$ protein signaling 5 , a protein upregulated in a variety of cancers, ${ }^{2}$ could block tumor-induced vascular remodeling and enhance delivery of therapeutics. ${ }^{3}$

In the new study by Ruth Ganss of the Western Australian Institute for Medical Research and colleagues, the researchers knocked out regulator of G protein signaling 5 (Rgs5) in a murine model of pancreatic islet cell carcinogenesis, a model previously used in her lab that had shown overexpression of Rgs5 in the tumor vasculature, specifically in pericytes in the vascular bed. ${ }^{4}$ Pancreatic tumors in the resulting Rgs5-deficient mice had significantly improved oxygenation and significantly less vascular leakiness compared with tumors in wild-type mice $(p=0.008)$.

However, improved tumor vasculature is a double-edged sword. The Rgs5-deficient mice had greater tumor burden and lower survival compared with their wild-type counterparts. Yet, in another set of experiments described in the Nature paper, Ganss' group observed something new-improved tumor vasculature created an influx of immune cells into the tumor.

In mice that received preactivated tumor antigen-specific $\mathrm{T}$ cells, Rgs5-deficient mice had significantly higher levels of $\mathrm{CD} 8^{+} \mathrm{T}$ cell infiltration into their tumors compared with those seen in wild-type mice $(p<0.0001)$. Moreover, the Rgs5-deficient mice showed significantly prolonged survival following $\mathrm{T}$ cell transfer compared with that of wild-type mice ( $p=0.0004$ ).

The goal of this strategy is to remodel or normalize tumor vessels and thus enhance influx of immune cells, with subsequent retardation of tumor growth, Ganss told SciBX. The goal of knocking out Rgs5 "is not to kill tumor vessels," she noted, which would ultimately increase hypoxia and necrosis and decrease perfusion and drug or immune cell penetration.

Ganss, who is associate professor and principal research fellow at the institute, noted that that Rakesh Jain and colleagues at Harvard Medical School observed a similar "tumor vessel normalization phase" following treatment of solid tumors with the anti-VEGF antibody Avastin bevacizumab.

Avastin, a humanized $\mathrm{mAb}$ against VEGF from Genentech Inc. and Roche, is marketed to treat metastatic colorectal cancer, metastatic non-small cell lung cancer (NSCLC) and breast cancer.

Jain has hypothesized that angiogenesis inhibitors like Avastin reduce the population of leaky tumor vessels while leaving behind a subset of normalized vessels that can potentially open up tumors to penetration by chemotherapy. ${ }^{5,6}$ Jain is professor of radiation oncology at Harvard Medical School and director of the Steele Laboratory at the Massachusetts General Hospital.

"The study by Ganss et al. reveals another mechanism by which tumor vasculature can be targeted with a resulting vascular normalizing phenotype," said Dan Duda, a research colleague of Jain and assistant professor of radiation oncology at Massachusetts General Hospital and Harvard Medical School whose research focuses on tumor vasculature and the tumor microenvironment. "The novelty here is that Rgs5 is expressed primarily in pericytes, not in vascular endothelial cells, which are the targets of antiangiogenic drugs like Avastin and Sutent."

Sutent sunitinib, a small molecule from Pfizer Inc. that inhibits multiple receptor tyrosine kinases, is marketed to treat gastrointestinal stromal tumors (GIST) and advanced renal cell carcinoma (RCC).

In healthy tissue, blood vessel walls are lined by a layer of endothelial cells that are enveloped by a secondary layer of cells called pericytes, which help maintain the vessel's structural integrity. In tumor tissue, blood vessels are typically covered by fewer pericytes, which can contribute to decreased vessel stability and perhaps increased leakiness.

The Nature authors wrote that "whereas VEGF-blocking therapies clearly enhance drug efficacy, correlating with changes in vascular morphology, pericyte maturation shown in this study may also contribute to the anti-tumor effects by directly influencing leukocyte attachment and transmigration into tumor parenchyma."

They also noted that some tumors become resistant to VEGF drugs and the mechanism involving Rgs5 could potentially reverse "tumor angiogenesis independently of anti-angiogenic drugs by targeting Gprotein signaling."

Duda provided a slightly different rationale for a combined attack.

"As the Nature paper makes clear, targeting Rgs5 by itself will probably have little, if any, antitumor efficacy, since you're actually improving the function of tumor blood vessels without restricting their formation," he told SciBX. "Thus, much like the case with the anti-VEGF antibody Avastin, optimal targeting of Rgs5 would very likely occur in the context of a combination with an efficient cytotoxic therapy." 


\section{TARGETS \& MECHANISMS}

He added: "I would really like to see further preclinical work that explores Rgs5 inhibition in combination with radiation therapy or chemotherapy."

Kristen Hege, VP of clinical affairs at Cell Genesys Inc., told SciBX that "combining Rgs5 inhibition with immunotherapy certainly makes sense" and that "targeting tumor vasculature can enhance migration of immune effector cells into the tumor and thus potentially improve cancer vaccine efficacy."

Indeed, in the Nature paper, Rgs5-deficient mice that were treated with a vaccine consisting of pancreatic tumor antigen and immunostimulatory $\mathrm{CpG}$ oligodeoxynucleotide 1668 had significantly higher survival than that of wild-type mice that were vaccinated $(p<0.0001)$.

Cell Genesys' GVAX immunotherapy for pancreatic cancer is in Phase II testing to treat pancreatic cancer. The whole-cell vaccine consists of two allogeneic pancreatic tumor lines that are engineered to secrete granulocyte/macrophage colony-stimulating factor (GM-CSF) to recruit antigen presenting cells (APCs). In a Phase I trial, the vaccine elicited $\mathrm{CD}^{+} \mathrm{T}$ cells specific for mesothelin, which is upregulated in many pancreatic cancers. ${ }^{8}$

Cell Genesys' most advanced product, GVAX vaccine for prostate cancer, has completed Phase II testing to treat early stage disease.

According to Hege, "further characterization of Rgs5's role in pancreatic cancer ought to include preclinical models with more malignant and/or metastatic variants of disease. This would help clarify the extent to which the observed effects are general to pancreatic cancer or more specifically related to the insulinoma model used in the Nature paper."

According to Ganss, the insulinoma model was established in 1985 by Doug Hanahan and has since been used as a platform for studying antiangiogenesis therapies and immunotherapies, and "its clinical relevance as a multistep cancer model has been widely accepted."

Hanahan and colleagues at the University of California, San Francisco have used the model to identify and study vascular markers that are specific to different stages of tumor progression. ${ }^{9}$

Ganss also told SciBX that future work will study how vessel normalization in an Rgs5-deficient tumor environment affects penetration of cytotoxic drugs and immune effector cells. She said her lab will continue to use Rgs5 knockout models to investigate "what signaling pathways Rgs5 controls in the tumor environment and how Rgs5 itself is regulated."
In addition, Ganss noted that "if we find that Rgs5 regulates a defined pathway, targeting other molecules of the signaling cascade might also be feasible."

Preclinical research is ongoing in a number of academic labs to identify and design inhibitors of the Rgs family of proteins, though primarily in CNS indications. ${ }^{10,11}$

"This link between Rgs proteins and cancer is newly appreciated. Only very recently have gene knockout animals become available that allow study of the roles of Rgs proteins in whole-animal physiology," John Hepler told SciBX.

Hepler is associate professor of pharmacology at Emory University. His research partly focuses on the role of Rgs proteins in cell signaling pathways and neurotransmission.

"Many Rgs proteins are in theory desirable drug targets because, like the $G$ protein-coupled receptors they modulate, Rgs proteins are expressed discretely in specific cells and tissues, which optimizes therapeutic targeting potential and minimizes cross-tissue side effects," said Hepler.

\section{REFERENCES}

1. Fulmer, T. SciBX 1(1), 9-10; Jan 31, 2008

2. Boss, C. et al. Clin. Cancer Res. 13, 3347-3355 (2007)

3. Hamzah, J. et al. Nature; published online April 16, 2008; doi:10.1038/ nature06868

Contact: Ruth Ganss, Western Institute for Medical Research, UWA Centre for Medical Research, Perth, Australia e-mail: ganss@waimr.uwa.edu.au

4. Berger, M. et al. Blood 105, 1094-1101 (2005)

5. Willett, C. et al. Nat. Med. 2, 145-147 (2004)

6. Jain, R. Science 307, 58-62 (2005)

7. Bergers, G. \& Song, S. Neuro. Oncol. 7, 452-464 (2005)

8. Thomas, A. et al. J. Exp. Med. 200, 297-306 (2004)

9. Joyce, J. et al. Cancer Cell 4, 393-403 (2003)

10. Neubig, R. \& Siderovski, D. Nat. Rev. Drug Discov. 1, 187-196 (2002)

11. Hepler, J. Mol. Pharmacol. 64, 547-549 (2003)

\section{COMPANIES AND INSTITUTIONS MENTIONED}

Cell Genesys Inc. (NASDAQ:CEGE), South San Francisco, Calif.

Emory University, Atlanta, Ga.

Genentech Inc. (NYSE:DNA), South San Francisco, Calif.

Harvard Medical School, Boston, Mass.

Massachusetts General Hospital, Boston, Mass.

Pfizer Inc. (NYSE:PFE), New York, N.Y.

Roche (SWX:ROG), Basel, Switzerland

University of California, San Francisco, Calif.

Western Australian Institute for Medical Research, UWA Centre for Medical Research, Perth, Australia 